\title{
REPRESENTATIVE LOAD SEQUENCE FOR THE PZL-130 ORLIK
}

\author{
Andrzej Leski \\ Lukasz Obrycki
}

\author{
Air Force Institute of Technology, Warsaw, Poland
}

\section{INTRODUCTION}

Variable loads affect an aircraft throughout its operation. Some of them are determined by maneuver loads others are random eg. gust loads. Each particular flight has its own load sequence. Two equivalent flights (the same mission) performed by the same pilot during similar weather conditions are different. The load sequence acting on a structure during flight is recorded by an onboard flight recorder. An increase in the recorder accuracy emphasizes the differences between flights. Originally, the only parameter used to perform fatigue load assessment was the flight time. In this case it is easy to show similarity between flights. Since nz-cycle recorders were applied the flight loads have been monitored more precisely. The clear view on the flight loads is possible if the aircraft is equipped with a multi-channel flight data recorder. The digital flight data recorder can store the load history, which is very important for fatigue calculation. The load history informs of the load cycle sequence and a retardation model in fatigue calculation can be applied.

The whole usage history of aircraft is hard to collect because of the lack of data. Even if all the data were collected it would be impractical to use them because of a large number of data collected for each aircraft during its service life. If the load history consists of such a large number of data, it cannot be used either for numerical calculation or laboratory fatigue tests. The representative load sequence for a selected aircraft or helicopter population has been an area of interest for scientists for several years as well as the topic of scientific projects. Among the developed representative load sequence are:

- Falstaff

- Turbistan

- Helix

- Felix

A representative load sequence can be used for fatigue calculation and for crack growth analysis, which are performed for aircraft structural integrity purposes. This sequence can also be used for a full scale fatigue test or for laboratory tests of aircraft parts or specimens. These activities support aircraft reliability and safety.

\section{REPRESENTATIVE LOAD SEQUENCE FOR THE PZL-130 ORLIK}

The aim of developing the representative load sequence (RLS) for the PZL-130 Orlik was to obtain a load time history which was the best approximation of loads acting on the aircraft structure during average flight. This load time history should be short enough to be used for numerical calculation or laboratory tests. Good approximation can be achieved if a large number of performed flights were taken into consideration. Such a large number of recorded flights can be collected during long operational usage. Otherwise good approximation can be assessed based on 
an extensive flight test program. Developing RLS is an extraction of crucial (for fatigue and crack growth analyses) information from the collected data. The information which must be retained in RSL is:

- number of fatigue cycles of particular amplitude and mean value,

- cycle sequence.

The data base of PZL-130 Orlik consists of more than 35000 collected downloads (each download comprises one flight). Because PZL-130 Orlik is a trainer, all performed flights were training missions. All collected downloads have available information about an exercise number which was performed by the pilot. This way statistics for exercise frequency can be calculated.

At the beginning of developing the representative load sequence for PZL-130 Orlik the following assumptions have been made:

a) Flight loads can be characterized by Nz-cycle;

b) RLS would consists of 100-150 flight sequence;

c) Flights selected for RLS should match the statistics for exercise frequency;

d) Nz-cycle statistics for RLS should mach nz-cycle statistics for all collected flights.

The first two assumptions determine the general way in which the RLS should be developed. The RLS should be time series of nz-signal taken from selected flights. The main difficulty in achieving a good representative lies in the right choice of flights.

The assumption c) depends on how the load sequence is prepared. This sequence should include flights from a variety of exercises. The number of flights of a certain number of exercises must correspond to the statistics for the exercise frequency. Because the RLS would consists of 100-150 flight sequence, some of exercises would not be represented (the number of exercises in the collected data base is greater than 100). The decision whether an exercise is selected or not is taken on the basis of similarity of the mean load profile for the exercise with the mean load profile for all collected flights. An exercise whose profile differs significantly from the mean profile of all collected flights should be represented in RLS.

The assumption d) is crucial for the representativeness of RLS. The mean number of cycles for each amplitude must be the same for RLS as for all collected flights.

\section{THE ALGORITHM OF OPTIMAL FLIGHT SELECTION 3.1. Definitions and indications}

The flight selection for RLS is an optimization task. The aim function which should be minimized is the similarity of load profiles for RLS and the mean load profile (for all collected flights). The algorithm of optimal flight selection in mathematical notation is presented below.

$\mathbf{V}_{i}^{k}$ - vector corresponding to the one flight from the collected data base,

$k$ - number of exercise for flight

$i$ - index of flight (flight number)

Where:

$$
k \in[1,2, \ldots, i k]
$$

$i k$ - number of exercises represented in RLS.

$$
i \in\left[1,2, \ldots, i i_{k}\right]
$$

Where:

$i i_{k}$ - number of collected flights for exercise no. $k$. 
Components of vector $\mathbf{V}_{i}^{k}$ are the number of cycles of a particular amplitude recorded in a flight (standardized to the 1000 hours flight).

$$
\mathbf{V}_{i}^{k}=\left[v_{1}, v_{2}, v_{2}, \ldots, v_{n}\right]
$$

Where:

$v_{n}$ - the number of nz-cycles of a particular amplitude recorded in a flight (standardized)

The standardization procedure is shown below:

$$
v_{n}=\frac{l_{n z=n}}{t_{i}} 1000
$$

Where:

$l_{n z=n}$ - the number of nz-cycles of amplitude $\mathrm{n}$ in flight $\mathrm{i}$, $t_{i}$ - duration of flight i (in hours)

The mean load profile for all collected flights is represented by vector $\mathbf{M}$

$$
\mathbf{M}=\left[m_{1}, m_{2}, m_{2}, \ldots, m_{n}\right]
$$

Where components of vector $\mathbf{M}$ are the mean number of cycles of a particular amplitude recorded in a flight (standardized to the 1000 flight hours).

The $\mathbf{S}$ matrix is a set of vectors $\mathbf{V}_{i}^{k}$ needed for RLS. A schema of the matrix structure is presented below:

$\mathbf{S}=\left\{\begin{array}{c}\mathbf{V}_{o}^{1} \\ \mathbf{V}_{p}^{1} \\ \vdots \\ \mathbf{V}_{r}^{1} \\ \mathbf{V}_{s}^{2} \\ \mathbf{V}_{t}^{2} \\ \vdots \\ \mathbf{V}_{u}^{i k}\end{array}\right\}$

$\mathbf{V}_{o}^{1}, \mathbf{V}_{p}^{1}, \mathbf{V}_{r}^{1}$ are flights chosen for exercise 1;

$\mathbf{V}_{s}^{2}, \mathbf{V}_{t}^{2}$, are flights chosen for exercise 2 ;

$\mathbf{V}_{u}^{i k}$ are flights chosen for exercise $i k .$.

The term "set of vectors $\mathbf{V}$ which satisfy the condition of the configuration of $\mathbf{S}$ matrix" implies that exercise numbers of vectors comprising matrix $\mathbf{S}$ fit to the statistic of exercise frequency. In other words for each vector $\mathbf{V}$ comprising matrix $\mathbf{S}$, the exercise number is determined before the optimization starts. 
The MS is mean value vector for RLS. Its components are mean values for the columns of matrix S:

$$
m s_{n}=\frac{1}{i x m} \sum_{i x=1}^{i x m} v_{n}^{i x}
$$

Where:

ixm - number of flights comprising RLS.

The optimization task is to create matrix $\mathbf{S}$ from all available vectors $\mathbf{V}$ in such a way that vector MS is equal to vector M. In real optimization, the equality of the vectors cannot be achieved exactly so the difference has to be minimized. The difference between the vectors can be calculated by means of various norms. In this algorithm, the popular formula was used:

$$
\|\mathbf{M S}-\mathbf{M}\|=\sqrt{\sum_{i=1}^{i=n}\left(m s_{i}-m_{i}\right)^{2}}
$$

The vector of the difference MS-M is calculated using the formula:

$$
\mathbf{M S}-\mathbf{M}=\left[m s_{1}-m_{1}, m s_{2}-m_{2}, \ldots, m s_{n}-m_{n},\right]
$$

In similar way, other vectors of the difference are calculated.

\subsection{The Algorithm}

The algorithm of flights selection for representative load sequence is presented below:

1. Random selection of vectors $\mathbf{V}$ which satisfy the condition of the $\mathbf{S}$ matrix configuration, Calculation of vector MS components (7),

2. Calculation of difference vector $\mathbf{R}$ (between vectors $\mathbf{M S}$ and $\mathbf{M}$ ) (9),

3. Calculation of distance d1 between vectors $\mathbf{M S}$ and $\mathbf{M}$ (the norm of $\mathbf{R}$ ) (8);

4. Random selection of exercise number opt

5. For all vectors $\mathrm{V}$ from $\mathrm{S}$ matrix with the exercise number opt calculate the difference vector RX (between vectors RX and MS)

Where:

$$
\mathbf{R X} \mathbf{X}_{p}=\mathbf{M S}-\mathbf{V}_{p}^{o p t}
$$

$\mathbf{V}_{p}^{\text {opt }}$ - are components of $\mathbf{S}$ matrix.

6. Calculate similarity $\mathrm{C}_{\mathrm{p}}$ between difference vector $\mathbf{R X}$ and vector $\mathbf{R}$,

$$
C_{p}=\frac{\mathbf{R} \mathbf{X}_{p} \bullet \mathbf{R}}{\left\|\mathbf{R X}_{p}\right\|\|\mathbf{R}\|}
$$

Where the dot in the numerator above is the scalar product operator. 
7. Vector $\mathbf{V}_{p}^{\text {opt }}$ with the smallest $\mathrm{C}_{\mathrm{p}}$ value is chosen to be removed from matrix $\mathbf{S}$ because it causes the large increase of $\mathrm{d} 1$ value among other $\mathbf{V}$ vectors with exercise number opt. It should be noted that the selection of a particular vector to be removed depends on other $\mathbf{V}$ vectors with the same exercise number. This process assesses the compatibility between a particular vector and other vectors comprising matrix $\mathbf{S}$. If the compatibility between a particular vector and vector $\mathbf{M}$ was taken into account, it would result in eliminating from matrix $\mathbf{S}$ those vectors $\mathbf{V}$ which are not similar to vector $\mathbf{M}$. As a result, the final RLS would consist only of vectors $\mathbf{V}$ (flights) similar to the $\mathbf{M}$ vector (mean profile), which is not an intention of the RLS development.

8. The vector to replace the chosen one comes from other vectors (collected flights) with exercise number opt which are not part of matrix $\mathbf{S}$. For those vectors, distance values $\mathrm{C}_{\mathrm{r}}$ are calculated (like $C_{p}$ value in step 7). Among all vectors with exercise number opt one with the maximum $\mathrm{C}_{\mathrm{r}}$ value is chosen.

9. Before the vector replacement, the condition (12) is checked:

$$
\mathrm{d} 1^{\text {new }}<\mathrm{d} 1^{\text {old }}
$$

where:

$\mathrm{d} 1^{\text {new }}-$ is $\mathrm{d} 1$ value for matrix $\mathbf{S}$ after vector replacement

$\mathrm{d} 1^{\text {old }}-$ is $\mathrm{d} 1$ value for matrix $\mathbf{S}$ before vector replacement

If the condition (12) is not satisfied, it means that the replacement would not improve the solution and it should not be performed.

10. After the replacement, the new matrix $\mathbf{S}$ is created and the procedure can start once again. Steps 2-10 are repeated.

11. After several repetitions, the algorithm reaches a local minimum. Special techniques can be used to avoid local minimums e.g. generic algorithm. In this example the simplest technique has been chosen. The whole process is repeated several times. Because the algorithm starts with random initial data, it usually ends in different minimum. The best solution is taken as the final result.

\section{EXAMPLE}

In this example, the algorithm of optimal flight selection, described above, was tested for some prepared data. In table 1 data for 30 flights are presented (each row is one flight). The data for each flight consist of:

- $\quad$ exercise number (second column) - integer between 1 and 6

- numbers of nz-cycles (five amplitude levels)

Not all data used were real data. Some were prepared for the test purpose only.

The assumption was made that RLS should consist of:

- 3 flight for exercise no 1

- 2 flight for exercise no 2

- 2 flight for exercise no 3

- 1 flight for exercise no 4

- 1 flight for exercise no 5

- 1 flight for exercise no 6

Additionally, vector $\mathbf{M}$ was the mean value of all 30 vectors $\mathbf{V}$. In this example, the vector $\mathbf{M}$ components were:

$\mathbf{M}=[2.1,1.73,1.13,1.267,1.567]$ 
Tab 1. Data for the example 1

\begin{tabular}{|c|c|c|c|c|c|c|}
\hline $\begin{array}{c}\text { Flight } \\
\text { number }\end{array}$ & $\begin{array}{l}\text { Exercise } \\
\text { number }\end{array}$ & $\begin{array}{c}\text { Ilz-cycle } \\
(n z=1) \\
\text { number }\end{array}$ & $\begin{array}{c}\text { Ilz-cycle } \\
(n z=2) \\
\text { number }\end{array}$ & $\begin{array}{c}\text { Irz-cycle } \\
(\mathrm{nz}=3) \\
\text { number }\end{array}$ & $\begin{array}{c}\text { Irz-cycle } \\
(\mathrm{nz}=4) \\
\text { number }\end{array}$ & $\begin{array}{c}\text { Irz-cycle } \\
(\mathrm{nz}=5) \\
\text { number }\end{array}$ \\
\hline 1 & 1 & 1 & 2 & 0 & 2 & 1 \\
\hline 2 & 1 & 2 & 1 & 1 & 1 & 1 \\
\hline 3 & 1 & 1 & 2 & 1 & 0 & 2 \\
\hline 4 & 1 & 3 & 1 & 0 & 1 & 1 \\
\hline 5 & 1 & 2 & 2 & 1 & 2 & 4 \\
\hline 6 & 1 & 1 & 3 & 1 & 1 & 1 \\
\hline 7 & 1 & 5 & 2 & 3 & 2 & 1 \\
\hline 8 & 1 & 3 & 1 & 0 & 1 & 2 \\
\hline 9 & 1 & 1 & 2 & 1 & 2 & 1 \\
\hline 10 & 1 & 4 & 1 & 3 & 1 & 1 \\
\hline 11 & 2 & 1 & 2 & 1 & 1 & 1 \\
\hline 12 & 2 & 2 & 4 & 0 & 2 & 2 \\
\hline 13 & 2 & 1 & 2 & 1 & 0 & 1 \\
\hline 14 & 2 & 2 & 1 & 1 & 1 & 1 \\
\hline 15 & 2 & 2 & 2 & 1 & 1 & 3 \\
\hline 16 & 3 & 1 & 3 & 0 & 1 & 2 \\
\hline 17 & 3 & 3 & 2 & 1 & 2 & 1 \\
\hline 18 & 3 & 2 & 1 & 3 & 1 & 1 \\
\hline 19 & 3 & 3 & 2 & 1 & 1 & 3 \\
\hline 20 & 3 & 1 & 1 & 0 & 2 & 2 \\
\hline 21 & 4 & 3 & 1 & 1 & 1 & 1 \\
\hline 22 & 4 & 1 & 2 & 1 & 1 & 3 \\
\hline 23 & 4 & 2 & 1 & 0 & 2 & 1 \\
\hline 24 & 5 & 1 & 2 & 1 & 1 & 1 \\
\hline 25 & 5 & 4 & 1 & 1 & 1 & 2 \\
\hline 26 & 5 & 3 & 2 & 1 & 2 & 1 \\
\hline 27 & 5 & 1 & 1 & 4 & 1 & 1 \\
\hline 28 & 6 & 3 & 2 & 0 & 2 & 2 \\
\hline 29 & 6 & 1 & 1 & 1 & 1 & 1 \\
\hline 30 & 6 & 3 & 2 & 4 & 1 & 2 \\
\hline
\end{tabular}

At the beginning matrix $\mathbf{S}$ was filled with the random set of vectors $\mathbf{V}$

$$
\mathbf{S}=\left\{\begin{array}{c}
\mathbf{V}_{1}^{\mathbf{1}} \\
\mathbf{V}_{8}^{\mathbf{1}} \\
\mathbf{V}_{3}^{\mathbf{1}} \\
\mathbf{V}_{14}^{2} \\
\mathbf{V}_{11}^{2} \\
\mathbf{V}_{17}^{3} \\
\mathbf{V}_{19}^{3} \\
\mathbf{V}_{23}^{4} \\
\mathbf{V}_{26}^{5} \\
\mathbf{V}_{29}^{6}
\end{array}\right\}
$$

Vector MS was calculated for matrix $\mathbf{S}$ :

$\mathbf{M S}=[2,1.6,0.7,1.3,1.4]$

Then the difference vector $\mathrm{R}$ was calculated (9)

$\mathbf{R}=[-0.1,-0.133,-0.433,0.033,-0.167]$ 
The distance d1 for vector $\mathbf{R}$ was

$$
\mathrm{d} 1=0.9309
$$

During the first iteration the exercise number 4 was randomly selected for optimization.

$$
\text { opt }=4
$$

Because:

In matrix $\mathbf{S}$ there was only one vector $\left(\mathbf{V}_{23}^{4}\right)$ for exercise number 4 thus it instantly became the candidate for the replacement. Then $\mathrm{C}$ values were calculated for all available vectors for exercise number 4 (vectors $\mathbf{V}_{21}^{4}$ and $\mathbf{V}_{22}^{4}$ ).

$$
\begin{aligned}
& \mathrm{C}_{21}=0.1448 \\
& \mathrm{C}_{22}=0.3687
\end{aligned}
$$

Maximum $\mathrm{C}$ value was for vector $\mathbf{V}_{21}^{4}$

After the first iteration the new value of distance $\mathrm{d} 1$ was

$$
\mathrm{d} 1=0.8165
$$

Because

$\mathrm{d} 11^{\text {new }}<\mathrm{d} 1^{\text {old }}$

then the vector $\mathbf{V}_{22}^{4}$ replaced the vector $\mathbf{V}_{23}^{4}$ in matrix $\mathbf{S}$

Matrix $\mathbf{S}$ after first iteration was:

$$
\mathbf{S}=\left\{\begin{array}{c}
\mathbf{V}_{1}^{\mathbf{1}} \\
\mathbf{V}_{8}^{\mathbf{1}} \\
\mathbf{V}_{3}^{\mathbf{1}} \\
\mathbf{V}_{14}^{2} \\
\mathbf{V}_{11}^{2} \\
\mathbf{V}_{17}^{3} \\
\mathbf{V}_{19}^{3} \\
\mathbf{V}_{22}^{4} \\
\mathbf{V}_{26}^{5} \\
\mathbf{V}_{29}^{6}
\end{array}\right\}
$$

During the second iteration the exercise number 3 was randomly selected for optimization.

$$
\text { opt }=3
$$

Because there were two vectors $\left(\mathbf{V}_{17}^{3}, \mathbf{V}_{19}^{3}\right)$ in matrix $\mathbf{S}$ then the $\mathrm{C}$ parameter was calculated for both vectors.

$$
\begin{aligned}
& \mathrm{C}_{17}=0.6089 \\
& \mathrm{C}_{19}=0.3260
\end{aligned}
$$

The minimum $\mathrm{C}$ value was for vector $\mathbf{V}_{19}^{3}$ and this vector was selected for replacement.

Then the $\mathrm{C}$ values were calculated for all available vectors for exercise number 3 .

$$
\begin{aligned}
& C_{16}=-0.5923 \\
& C_{18}=0.7741 \\
& C_{20}=-0.6540
\end{aligned}
$$


The maximum value $\mathrm{C}_{18}$ was for vector $\mathbf{V}_{18}^{3}$. Then the result of the replacement was checked.

Because the new value $\mathrm{d} 1$ was greater than 0.8165 thus the replacement was not carried out.

During the third iteration the exercise number 6 was randomly selected for optimization.

$$
\mathrm{opt}=6
$$

As there is only one vector for exercise number 6 in matrix $\mathbf{S}$, it thus becomes the candidate for the replacement without additional calculations. Based on the $C$ values for other vectors $\left(\mathbf{V}_{28}^{6} \mathrm{i} \mathbf{V}_{30}^{6}\right)$ :

$$
\begin{aligned}
& C_{28}=-0.1317 \\
& C_{30}=0.0587
\end{aligned}
$$

vector $\mathbf{V}_{30}^{6}$ was selected for the replacement.

In this case, the new $\mathrm{d} 1(0.5477)$ value was smaller than the old one so vector $\mathbf{V}_{29}^{6}$ was replaced with vector $\mathbf{V}_{30}^{6}$ in the matrix $\mathbf{S}$.

The new $\mathbf{S}$ matrix was:

$$
\mathbf{S}=\left\{\begin{array}{c}
\mathbf{V}_{1}^{\mathbf{1}} \\
\mathbf{V}_{8}^{\mathbf{1}} \\
\mathbf{V}_{3}^{\mathbf{1}} \\
\mathbf{V}_{14}^{2} \\
\mathbf{V}_{11}^{2} \\
\mathbf{V}_{17}^{3} \\
\mathbf{V}_{19}^{3} \\
\mathbf{V}_{22}^{4} \\
\mathbf{V}_{26}^{5} \\
\mathbf{V}_{30}^{6}
\end{array}\right\}
$$

The algorithm reached a local minimum. The procedure was started many times with various initial data. The best solution reached was this presented below:

$$
\mathbf{S}=\left\{\begin{array}{c}
\mathbf{V}_{1}^{\mathbf{1}} \\
\mathbf{V}_{10}^{\mathbf{1}} \\
\mathbf{V}_{2}^{\mathbf{1}} \\
\mathbf{V}_{14}^{2} \\
\mathbf{V}_{12}^{2} \\
\mathbf{V}_{18}^{3} \\
\mathbf{V}_{19}^{3} \\
\mathbf{V}_{22}^{4} \\
\mathbf{V}_{26}^{5} \\
\mathbf{V}_{28}^{6}
\end{array}\right\}
$$

The d1 value was 0.4082 . 


\section{FINAL REMARKS}

The real RLS was obtained for the trainer PZL-130 Orlik using the presented above algorithm. 3353 recorded real flights were taken into consideration. The flights represented nine exercises. Vectors consisted of seven components. Nz-cycles were calculated for the amplitude levels: $0.5,1$, $1.5,2,2.5,3,3.5$. Numbers of cycles were normalized to $10 \mathrm{~h}$ of flight.

The differences between the vectors were calculated by means of "the fatigue formula". This formula took into account the influence of nz-cycle amplitude on fatigue damage. The formula is presented below and weights are showed in tab.3:

$$
\|\mathbf{R}\|=\sqrt{\sum_{i=1}^{i=n} w_{i}^{2}\left(m s_{i}-m_{i}\right)^{2}}
$$

Tab. 2. The weights

\begin{tabular}{|l|l|}
\hline Component Number & weight \\
\hline 1 & 0.25 \\
\hline 2 & 1 \\
\hline 3 & 2.25 \\
\hline 4 & 4 \\
\hline 5 & 6.25 \\
\hline 6 & 9 \\
\hline 7 & 12.25 \\
\hline
\end{tabular}

The calculated RLS can be used for numerical simulations of crack growth or for laboratory tests. The results of such analyses or tests have great value because RLS is very similar to real usage profile.

The presented algorithm can be also used for other similar problems even not connected with aircraft. 


\section{REFERENCES}

[1] Alten F. Grandt Jr, „Fundamentals of Structural Integrity. Damage Tolerant Design and Nondestructive Evaluation", Wiley, 2004.

[2] R.S. Rutledge. Evaluation, modification and damage tolerance of an in-service aircraft critical area. $25^{\text {th }}$ ICAF Symposium. Rotterdam 2009.

[3] G.M. van Dijk, J.B. de Jonge. Introduction to a fighter aircraft loading standard for fatigue evaluation "FALSTAFF". 8 ICAF Symposium, Lozanna 1975.

[4] P.R. Edwards, J. Darts. Standardised fatigue loading sequences for helicopter rotors. Part I. NLR, Amsterdam.

[5] P.R. Edwards, J. Darts. Standardised fatigue loading sequences for helicopter rotors. Part II. NLR, Amsterdam.

[6] M. Wrona. Wyznaczanie profilu średniego śmigłowców Mi-24. ITWL, Sprawozdanie nr 36/31/2008. Warszawa 2008.

[7] A.C. Buckner. F-16 Aircraft Structural Integrity Program (ASIP) Data Processing Training Modules - APVIb Delivery Order 0302. Lockheed Martin, Fort Worth 2006

[8] Prof. Grigory I. Nesterenko INVESTIGATION OF MATERIAL PROPERTIES DEGRADATION IN OPERATED AIRPLANES STRUCTURES 9th Joint FAA/DoD/NASA Aging Aircraft Conference.

[9] R.G. Forman i inni. Fatigue Crack Growth Database for Damage Tolerance Analysis. FAA, Waszyngton 2005.

[10] Introduction to NASGRO practical use and advanced techniques. Southwest Research Institute, San Antonio 2008.

[11] S. Klimaszewski i inni. Opracowanie widm obciążeń dla skrzydła samolotu Su-22. ITWL, Sprawozdanie nr 66/31/2005, Warszawa 2005. 